\title{
Dinâmica reprodutiva de Salminus brasiliensis (Cuvier, 1816) no Pantanal
}

A captura de Salminus brasiliensis (Cuvier, 1816) no Pantanal está proibida desde 2012 com o intuito de preservação da espécie, considerando que esta medida proporcione o reestabelecimento do seu nível populacional. O Pantanal é considerado um ambiente propício para a manutenção ictiofauna, sendo composto por diferentes fitofisionomias e riqueza de habitat, o que pode promover uma efetiva recuperação da espécie, principalmente em áreas de alto grau de preservação, como é o caso de Estações Ecológicas. Assim, este estudo analisou a dinâmica reprodutiva de S. brasiliensis, popularmente conhecido como dourado, entre os anos de 2004 a 2017, coletados na Estação Ecológica de Taiamã e rio Sepotuba no estado de Mato Grosso, analisando 123 espécimes (74 fêmeas e 49 machos). 0 maior número de machos e fêmeas estão contidos nos intervalos de classes de 57,05 a 62,66 e 62,66 a $68,27 \mathrm{~cm}$, respectivamente. Estes resultados indicam que os espécimes coletados nessa região possuem comprimento total maiores do que os citados na literatura. Já a reprodução da espécie está de acordo com outros estudos, em que o período de atividade reprodutiva ocorre entre a estiagem e enchente, cessando durante o período de águas altas. Embora o período reprodutivo esteja de acordo com outros estudos, ele difere daquele estabelecido pela lei estadual de Mato Grosso, por exemplo, o que pode implicar nas tomadas de decisão para a espécie estudada. As condições desta espécie no ambiente pantaneiro refletem a qualidade ambiental do sistema, apontando para a importância de pesquisas nessa área considerando a elaboração e implementação de políticas públicas e uso sustentável dos recursos naturais, bem como a valoração das áreas de proteção como repositório da ictiofauna.

\section{Reproductive dynamics of Salminus brasiliensis (Cuvier, 1816) in the Pantanal}

The capture of Salminus brasiliensis (Cuvier, 1816) in the Pantanal is prohibited since 2012 in order to preserve the species, considering that this decision provide the restoration of the population. The Pantanal is considered an important environment to the ichtyofauna preservation, presenting distinct phytofisiognomy and high habitat richness, which promote an efective species recuperation, mainly in areas os high level of preservation, such as Ecological Stations. Therefore, this study aimed to identify the reprodcutivity dynamics of S. Brasiliensis, popularly known as dourado between 2004 and 2017, sampled in the Taiamã Ecological Station and the Sepotuba river in the Mato Grosso state, analysing 123 specimens ( 74 males and 49 females). The highest number of males and females are between the class interval of 57,05 to 62,66 for males and 62,66 to 68,27 for females. These results indicate that the specimens sampled in the region have the total lenght higher than others cited in the literature. However, the reproduction dynamics is accoring to other studies, where the period of reproduction is between the dry and high water table. Although the reproduction is according to other studies, it differs from what is stablished in the law of the Mato Grosso state, for instance, which imply in a special atention to the decisions taken about the studied species. The conditions of this species in the Pantanal reflect the environmental quality, pointing out the importance of researches in this area considering the elaboration and implementation of public politics and sustenable uses of the natural resources, like the valuation of areas of total protection as ichthyofauna repository.

Keywords: Gonadosomatic Index; Hepatosomatic Index; Conservation Station; Sexual Proportion; Corporal Condition.

Topic: Planejamento, Gestão e Políticas Públicas Ambientais

Reviewed anonymously in the process of blind peer.
Received: 02/12/2018

Approved: 26/01/2019
Larissa Nayara Lima Silva (D)

Universidade do Estado de Mato Grosso, Brasil http://lattes.cnpq.br/1900114813439396

http://orcid.org/0000-0003-3140-6188

larissalima1507@hotmail.com

Priscila Campos Santos (D)

Universidade do Estado de Mato Grosso, Brasil http://lattes.cnpq.br/3312544039354251

http://orcid.org/0000-0002-6400-4129

pricampossantos@gmail.com

Francimayre Aparecida Pereira de Jesus (iD Universidade do Estado de Mato Grosso, Brasil http://lattes.cnpq.br/6642769846888600 http://orcid.org/0000-0002-1617-9725 francy21pereirajv@gmail.com

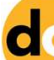

DOI: 10.6008/CBPC2179-6858.2019.001.0026
Ana Paula Dalbem Barbosa (iD)

Universidade do Estado de Mato Grosso, Brasil http://lattes.cnpq.br/4090241675573333 http://orcid.org/0000-0002-6282-4799 pauladalbem11@gmail.com

Ernandes Sobreira Oliveira Junior (iD)

Universidade do Estado de Mato Grosso, Brasil http://lattes.cnpq.br/7461346615427709

http://orcid.org/0000-0002-6953-6917

ernandes.sobreira@gmail.com

Claumir Cesar Muniz (D)

Universidade do Estado de Mato Grosso, Brasil

http://lattes.cnpq.br/2202899559144774

http://orcid.org/0000-0002-2082-2234

claumir@unemat.br

\section{Referencing this:}

SILVA, L. N. L.; SANTOS, P. C.; JESUS, F. A. P.; BARBOSA, A. P. D.; OLIVEIRA JUNIOR, E. S.; MUNIZ, C. C.; KANTEK, D. L. Z.. Dinâmica reprodutiva de Salminus brasiliensis (Cuvier, 1816) no Pantanal. Revista Ibero Americana de Ciências Ambientais, v.10, n.1, p.314321, 2019. DOI: http://doi.org/10.6008/CBPC2179-

Daniel Luiz Zanella Kantek iD

Universidade do Estado de Mato Grosso, Brasil http://orcid.org/0000-0001-9558-1503 daniel.kantek@gmail.com 


\section{INTRODUÇÃO}

Salminus brasiliensis (Cuvier, 1816) popularmente conhecido como dourado, é um peixe carnívoro de grande porte, podendo atingir mais de um metro de comprimento. Possui desova anual total, ovos semidensos, e não apresentam cuidado parental (VAZZOLLER, 1996). Entre os principais fatores que estabelecem a estratégia reprodutiva das espécies de peixes, destacam-se o dimorfismo sexual, tamanho dos ovos, fecundidade e período reprodutivo. Esses parâmetros podem ser considerados como a base para o estabelecimento dos principais padrões da história de vida de peixes (MAZZONI et al., 2006). A atividade reprodutiva possui relação com as reservas energéticas depositadas em diferentes partes do organismo a partir da ingestão de alimentos. O fígado e o peso das gônadas sofrem notáveis variações durante o ciclo reprodutivo (AGOSTINHO et al., 1990), devido principalmente ao processo de acumulo de material de reserva nos ovócitos em maturação (COSTA et al., 2005). Este tipo de informação pode ser utilizada como indicador das fases de desenvolvimento gonadal e na identificação das fases do ciclo (AGOSTINHO et al., 1990).

Para desenvolvimento de programas de monitoramento biológico, as comunidades de peixes possuem diversos benefícios como indicadores podendo disponibilizar informações sobre o ciclo de vida de grande número de espécies (ARAÚJO, 1998). Esta espécie é alvo de pescadores profissionais e amadores (ZANIBONI-FILHO et al., 2005). Entretanto, em todo o estado de Mato Grosso, a captura, a comercialização e o transporte desta espécie esta vedada desde 2012 de acordo com a lei estadual 9794, em seu artigo 17-A. Porém após o fechamento da pesca desta espécie nesta região poucos estudos foram desenvolvidos afim de fomentar sua compreensão ecológica e sua dinâmica reprodutiva. Assim, a pesquisa científica em torno dos aspectos ecológicos do dourado pode incrementar o conhecimento da espécie para que o manejo adequado seja empregado em virtude da reabertura da pesca deste. A importância de pesquisas nessa área se caracteriza como altamente relevante no que tange o manejo e uso sustentável dos recursos pesqueiros, o qual deve ser feito de forma planejada e racional (AQUINO, 2005).

O emprego de índices morfofisiológicos para a compreensão da dinâmica ecológica das espécies ictiológicas se remetem principalmente a utilização do índice hepatossomático, o qual relaciona o peso do peixe com o seu fígado, fornecendo uma resposta da qualidade ambiental (BRAGA et al., 2007); e o índice gonadossomático, utilizado para relacionar o tamanho do peixe com o peso da gônada, evidenciando seus estágios reprodutivos e a qualidade funcional dos órgãos reprodutivos, indicando as condições biológicas dos peixes e qualificando-os de acordo com o ambiente em que habitam, pois os índices refletem na condição do organismo (VAZZOLER, 1996). Desta forma, esse trabalho teve como objetivo descrever os índices de condição corporal, sendo o hepatossomático e o gonadossomático de Salminus brasiliensis, bem como descrever a dinâmica reprodutiva da espécie na região da Estação Ecológica de Taiamã e no rio Sepotuba. 


\section{MATERIAIS E MÉTODOS}

As coletas foram realizadas mensalmente em agosto de 2015 a dezembro de 2017 (autorização de número 51968-1 emitido pelo Sistema de Autorização e Informação em Biodiversidade - SISBIO), em três áreas distintas (Figura 1): leito do rio Paraguai (área da Estação Ecológica de Taiamã), área de campo (entorno da Estação Ecológica) e no rio Sepotuba (dados de 74 indivíduos coletados entre 2004 a 2011 disponibilizadas pelo Laboratório de Ictiologia do Pantanal do Norte - LIPAN da Universidade do Estado de Mato Grosso UNEMAT).

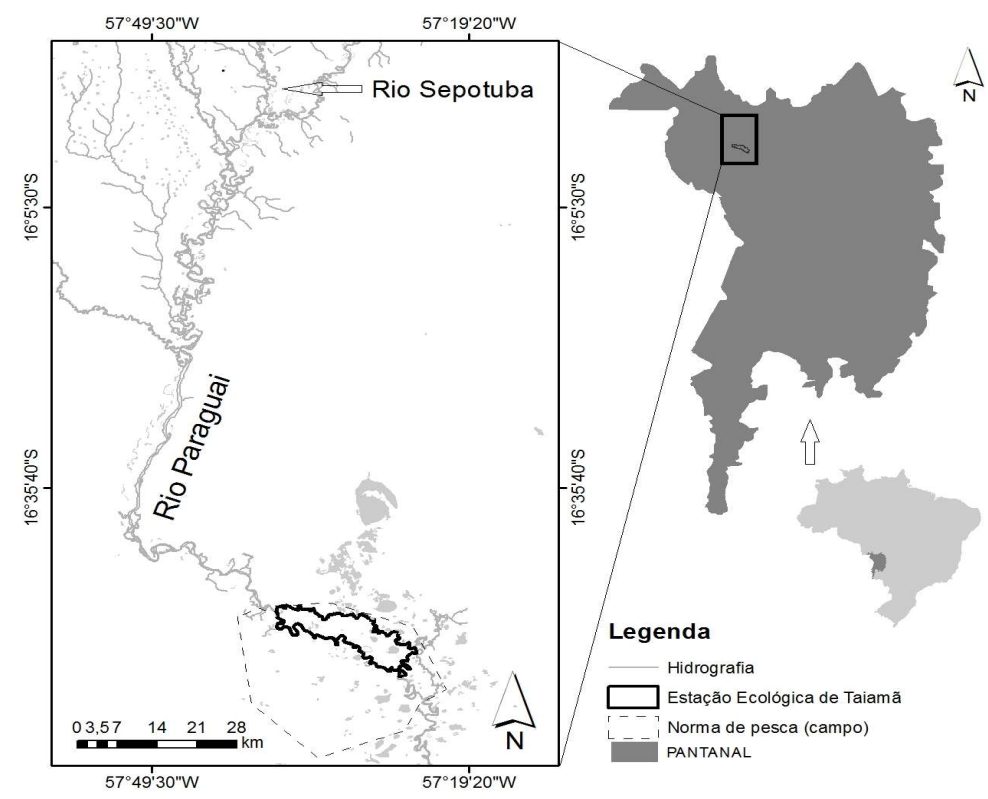

Figura 1: Área de estudo compreendendo confluência do rio Sepotuba com rio Paraguai e a Estação Ecologica de Taiamã em Cáceres/MT.

O método de Vazzoler (1996) foi utilizado para determinar a proporção sexual por classe de comprimento $(\mathrm{cm})$ e o período reprodutivo, com base em dois métodos para garantir a fidedignidade dos resultados: variação temporal da frequência de estágios de maturidade (utilizando a data da coleta, sexo e estágio de maturidade) e índice gonadossomático de acordo com Agostinho (1990) em que o IGS=100 (WG/W) na qual W representa a massa total e WG representa a massa dos ovários. Para o Índice Hepatossomático (IHS) foi adotado o método de Agostinho (1990), o qual é obtido a partir da relação a massa do fígado (g)/massa corporal total (g).

Os resultados foram dispostos de acordo com os meses do ano indicando os períodos hidrológicos de cheia (janeiro a março); vazante (abril a junho); estiagem (julho a setembro); e enchente (outubro a dezembro) de acordo com Resende (2008). Os dados morfofisiológicos dos peixes foram registrados em tabelas e analisados através de cálculos estatísticos através do pacote office (Excel 2016), com o nível de significância de $P<0,05$.

\section{RESULTADOS}

Os dados dos peixes coletados somados aqueles obtidos pelo banco de dados resultou em 123 indivíduos de S. brasiliensis analisados, sendo 74 fêmeas e 49 machos. Estes indivíduos variaram de $29 \mathrm{~cm}$ e 
$375 \mathrm{~g}$ a $85.1 \mathrm{~cm}$ a $9360 \mathrm{~g}$, os quais foram distribuídos em classes de comprimento (Figura 2). Para o mês de julho não houve captura de fêmeas.

O menor intervalo de classe para machos foi de 29 a 34,61 cm e o maior com 68,27 a 73,88 cm. Para fêmeas foi observado o menor intervalo de classe com 40,22 a 45,83 cm e o maior com 79,49 a 85,1. Entretanto, a maior frequência observada de machos e fêmeas foram nos intervalos de classe de 57,05 a $62,66 \mathrm{~cm}$ ( $\mathrm{n}=16$ espécimes) e 62,66 a 68,27 cm ( $\mathrm{n}=21$ espécimes), respectivamente.

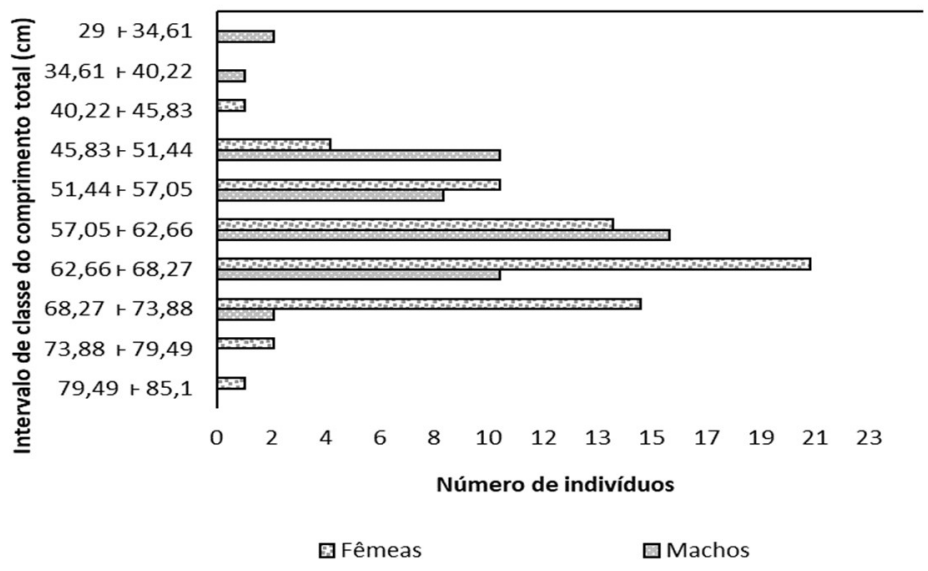

Figura 2: Abundância de S. brasiliensis de acordo com a proporção sexual apresentada por classe de comprimento total $(\mathrm{cm})$.

De acordo com a frequência dos estádios reprodutivos determinou-se o período reprodutivo da espécie em que a maioria dos indivíduos em estádio reprodutivo $A$ (repouso) $(34,48 \%)$, foram coletados no período de águas altas (janeiro a março). As gônadas em estádio de maturação ocorreram em maior percentagem $(87,50 \%)$ na enchente (outubro a dezembro). No estádio reprodutivo maduro foi observada maior frequência na estiagem e enchente, ambos com $40 \%$ e $100 \%$ das gônadas com o estádio reprodutivo esvaziado foram observados no período de cheia. Distinguindo machos e fêmeas os resultados demonstram que tanto machos quanto fêmeas iniciam a atividade reprodutiva no mês de agosto (estiagem), cessando o período reprodutivo nos meses de águas altas (Figura $3 \mathrm{~A}$ e B).
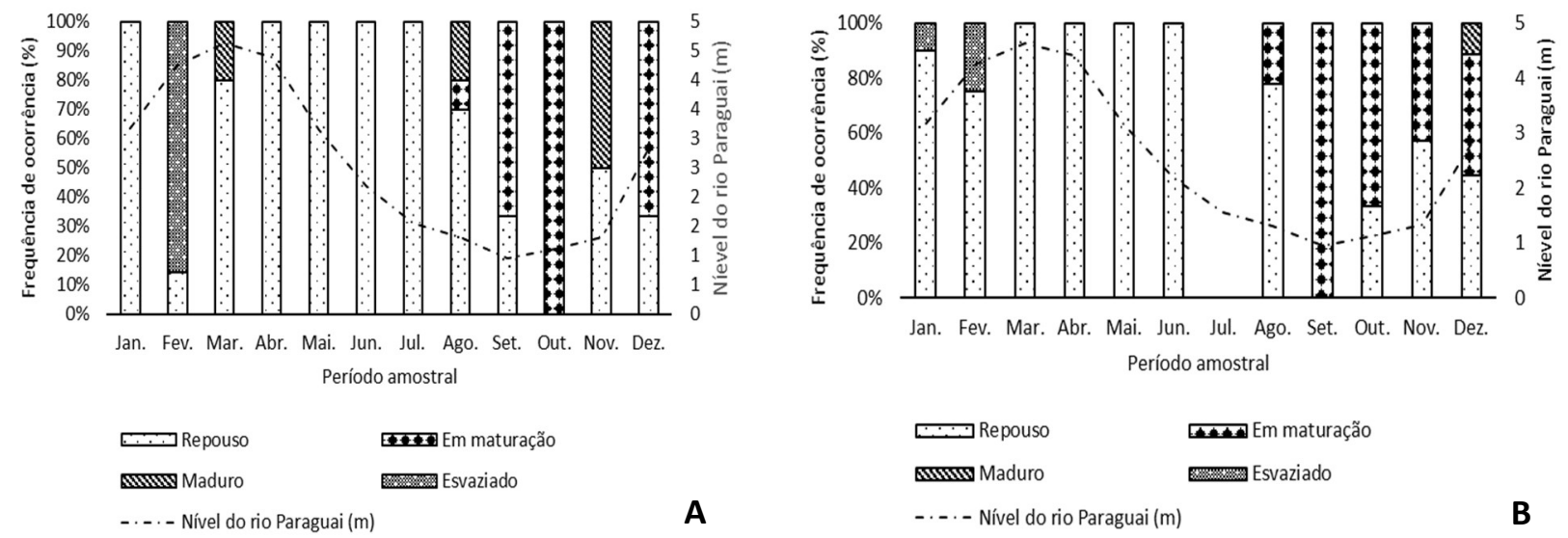

Figura 3: Estágios reprodutivos de Salminus brasiliensis para machos (A) e fêmeas (B) em relação aos meses de amostragem e o nível do rio Paraguai. 
Considerando todos os indivíduos capturados (machos e fêmeas), o índice gonadossomático (IGS) para a determinação do período reprodutivo se manteve com baixa variação na cheia, vazante e enchente. Já o maior índice hepatossomático observado foi no período de enchente (IHS=1,43). O IHS observado foi maior entre os períodos de cheia, vazante e estiagem (IHS=0,65,0,74 e 0,85, respectivamente) conforme Figura 4.
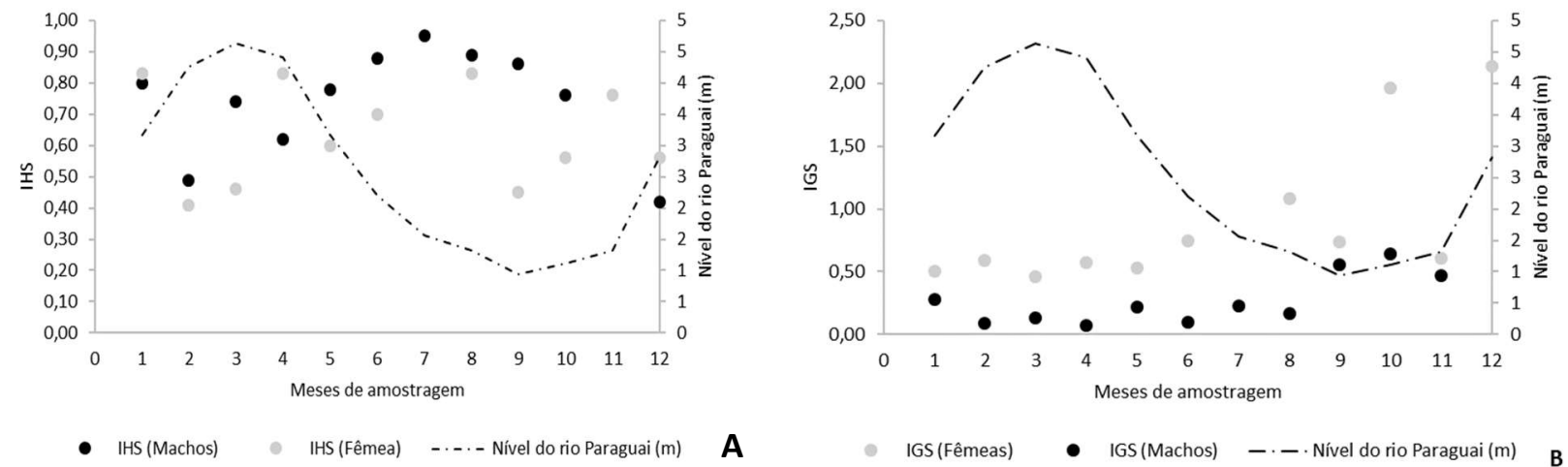

Figura 4: Índice gonadossomático (A) e hepatossomático (B) de Salminus brasiliensis de acordo com os meses de amostragem. Números indicam os meses do ano (1 a 12 = janeiro a dezembro).

A interação entre o índice gonadossomático com o hepatossomático demonstra uma relação negativa onde o aumento de um significa a redução do outro $\left(R^{2}=0,16, P<0,05\right)$. Porém, quando se diferencia os indivíduos entre machos e fêmeas, as relações se mostram positivias e significativas somente para o grupo dos machos $\left(R^{2}=0,19\right.$ para machos; e $R^{2}=0,02$ para fêmeas - Figura 5).
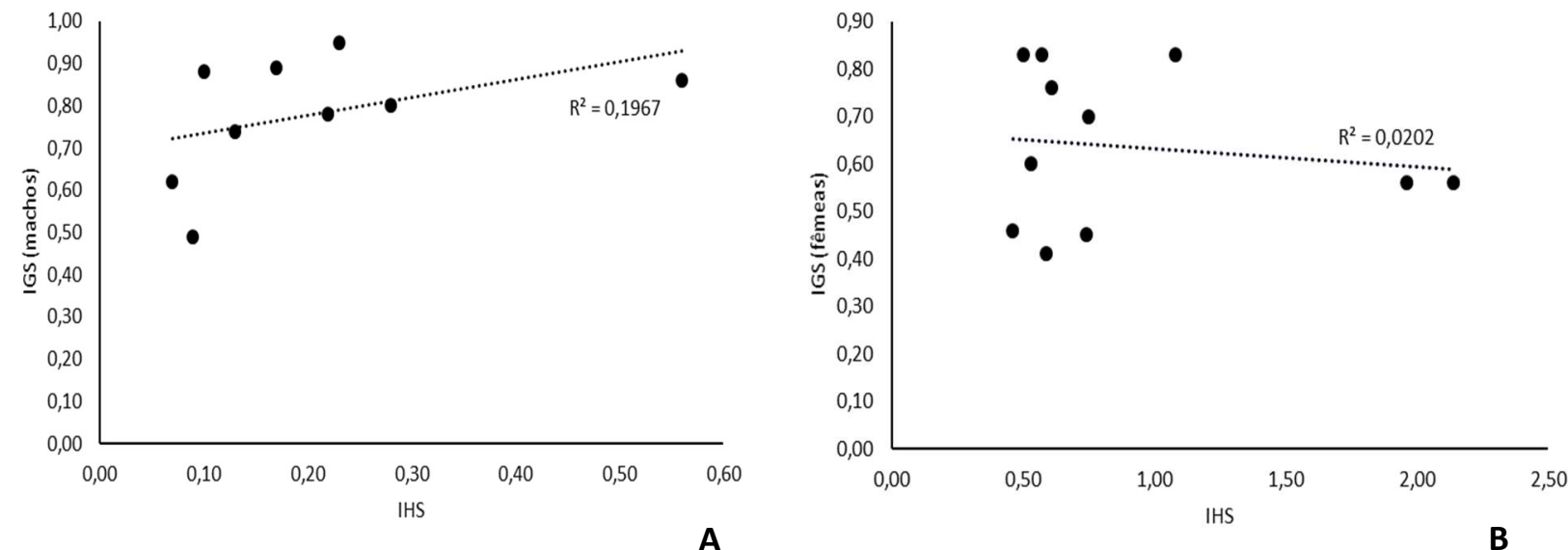

Figura 5: Relação linear entre os índices gonadossomático e hepatossomático de Salminus brasiliensis de acordo com os meses de amostragem separados por sexo (machos A; e fêmeas B).

\section{DISCUSSÃO}

Nossos resultados indicam que S. brasiliensis possui alta atividade reprodutiva, estando em atividade durante 8 meses do ano, somente no período de vazante e início da estiagem no Pantanal a espécie permanece em total repouso, não sendo encontrado qualquer indivíduo em estágio diferente (Figura 3). Este fato reflete na condição corpórea da espécie, a qual é maior durante o período de estiagem e cheia, tendo uma drástica redução durante o período de vazante (Figura 4). 
Foi observado que a maioria dos indivíduos machos apresentam comprimento total $(\mathrm{cm})$ nos menores intervalos de classes entre 29 a $57 \mathrm{~cm}$ e as fêmeas em maiores intervalos de classes de 62,66 a 85,1 $\mathrm{cm}$ de comprimento total (Figura 2), portanto, entre os indivíduos coletados os machos são menores que as fêmeas, o que condiz com outros estudos (MORAIS-FILHO et al., 1955; MOREIRA, 1999; MACHADO, 2003).

Porém, mesmo com os machos $(57,05$ a $62,66 \mathrm{~cm})$ estando distribuídos nos menores intervalos de classe e as fêmeas $(62,66$ a 68,27 cm) nos maiores, a variação desse intervalo é bem superior do que os encontrados na literatura, como no estudo de Honji (2007) que os maiores valores de comprimento total para machos foi de $35,5 \mathrm{~cm}$ e para fêmeas com $52,5 \mathrm{~cm}$.

Os índices de condição corporal são indicadores quantitativos do estado de bem-estar do peixe em seu ambiente, podendo apontar o seu período de desova (BRAGA, 1986). As espécies que realizam migrações reprodutivas consomem uma parcela das reservas energéticas somáticas podendo refletir em variações no fator de condição corporal (VAZZOLER, 1996). Neste estudo foi obtido maiores valores para o IHS em período que antecede a atividade reprodutiva (estiagem), correspondendo com Moreira (1999) em que para fêmeas obteve um aumento significativo na fase de maturação avançada. Segundo Moraes-Filho et al. (1955), Moreira (1999) e Braga et al. (1990) há uma redução natural na ingestão de alimentos durante a fase de maturação gonadal, justificando uma queda na condição corporal devido ao aumento das estruturas reprodutivas, neste estudo correlacionado principalmente para os indivíduos machos.

A condição corporal da espécie pode estar relacionada com os aspectos gonadossomáticos e hepatossomáticos, refletindo no seu comportamento ecológico (MOREIRA, 1999). De acordo com os resultados obtidos podemos arguir que o período da atividade reprodutiva e a proporção sexual entre machos e fêmeas condiz com que é relatado na literatura.

Determinar a proporção sexual é de grande importância para a caracterização da estrutura de uma população (VAZZOLER, 1996), e assim como esse estudo (com 74 fêmeas e 49 machos), existem diversos trabalhos realizados com o gênero Salminus em que se observa maior proporção sexual de fêmeas em comparação com os machos (OLARTE et al., 2006; BARBIERI et al., 2001; FEITOSA et al., 2004; VILLARES JUNIOR et al., 2007).

Para determinar o período reprodutivo foram utilizados dois métodos, de modo a obter uma maior fidedignidade dos resultados. Segundo Vazoller (1996), método que calcula a variação temporal da frequência de estádios de maturidade (Figura 3) é mais subjetivo por analisar os estágios reprodutivos das gônadas de forma macroscópica, de forma que o método que analisa o índice gonadossomático (Figura 4) é mais conciso por ser um indicador quantitativo. Portanto, os indivíduos machos e fêmeas apresentam suas gônadas em repouso no período de cheia.

As gônadas das espécimes em maturação na enchente e maduros entre a estiagem e enchente e na cheia estão com as gônadas esvaziadas. De acordo com o segundo método (Figura 4), o índice gonadossomático que avalia a relação entre o peso da gônada e o peso do corpo, o maior índice da IGS ocorre durante a enchente, indicando que este é o período mais ativo durante os estadios reprodutivos. Deste modo, de acordo com os resultados obtidos, o período reprodutivo do S. brasiliensis se inicia entre a estiagem 
e enchente com gônadas em maturação e maduras, indicando o período de desova estando esvaziadas na cheia. Resultados similares foram obtidos por diversos autores como Barbieri et al., (2001), Hermes-Silva (2003) e Machado (2003), em que o período reprodutivo inicia setembro com pico de maturação entre novembro a dezembro, correspondendo aos períodos de estiagem e enchente. Estes resultados confrontam o que é estabelecido na legislação matogrossense para o período de defeso (entre outubro e janeiro em Mato Grosso), o que pode resultar em consequências para S. brasiliensis em caso de liberação da atividade pesqueira.

O pico do índice hepatossomático ocorreu no período de estiagem quando ocorre a maturação das gônadas, com queda na enchente quando as gônadas apresentaram o estágio maduro (Figura 6). Segundo Bazzoli (1985), em algumas espécies o índice hepatossomático pode continuar diminuindo ao longo do período de desova e conforme Agostinho et al. (1990), o processo de reprodução e maturação leva a diminuição de reservas orgânicas da espécie, porém as modificações no fígado durante o desenvolvimento gonadal, tem sido associadas mais ao fornecimento de precursores vitelínicos do que à mobilização de reservas energéticas.

Nossos resultados também refletem na biologia da espécie no que tange a representatividade de machos e fêmeas. Por exemplo, foi observado que a maioria dos indivíduos machos apresentam comprimento total nos menores intervalos de classes e as fêmeas em maiores intervalos de classes. Mesmo com os machos estando distribuídos nos menores intervalos de classe e as fêmeas nos maiores, a variação desse intervalo é bem superior do que os encontrados na literatura, como no estudo de Honji (2007). Este fato indica o Pantanal como sendo um ambiente propício para o desenvolvimento desta espécie, podendo contribuir para sua conservação - espécie hoje protegida nos estados de Mato Grosso, Mato Grosso do Sul e Paraná.

\section{CONCLUSÕES}

Os resultados aqui apresentados refletem a importância de pesquisas nessa área, considerando a elaboração e implementação de políticas públicas e uso sustentável dos recursos naturais, buscando entender a manutenção do estoque pesqueiro, bem como a valoração das áreas de proteção integral como repositório íctiológico, mantendo o equilíbrio sócio ambiental no ambiente pantaneiro.

Estudos como estes indicam o papel das Unidades de Conservação na obtenção de dados científicos para o manejo e conservação de espécies da ictiofauna alvos de exploração recreativa e alimentação humana. Estas informações podem ser importantes para compor a base de dados dos períodos de defeso da piracema na Bacia do Alto Paraguai, bem como fornecer dados para outras políticas públicas, como a tomada de decisão relativa a captura da espécie nos próximo anos. Para esta espécie deve-se tomar especial atenção em relação ao período de defeso legalmente instituído, uma vez que sua atividade reprodutiva extrapola o que é definido por lei. 
AGRADECIMENTOS: Ao Instituto Chico Mendes de Conservação da Biodiversidade, por proporcionar a estrutura necessária para a coleta de dados e bolsa de estudos, a Universidade do Estado de Mato Grosso e o Laboratório de Ictiologia do Pantanal Norte pelo aporte necessário para a realização desse trabalho.

\section{REFERÊNCIAS}

AGOSTINHO, A. A.; BARBIERI, G.; VERANI, J. R.; HAHN, N. S.. Variação do fator de condição e do índice hepatossomático e suas relações com o ciclo reprodutivo em $R$ hinelepisaspera (Agassis, 1829) (Osteichthyes, Loricariidae) no Rio Paranapanema, Porecatu. Ciência e Cultura, v.42, p.711-714, 1990.

AQUINO, F. G.. Manejo e Uso dos Recursos Naturais. EMBRAPA, 2005.

ARAÚJO, F. G.. Adaptação do índice de integridade biótica usando a comunidade de peixes para o rio Paraíba do Sul. Rev. Brasil. Biol., v.58, p.547-558, 1998.

BARBIERI, G.; SALLES, F. A.; CESTAROLLI, M. A.. Reproductive and nutritional dynamics of Salminus maxillosus Valenciennes, 1849 (Pisces, Characidae) at Mogi Guaçu river, state of São Paulo, Brasil. Acta Scientiarum, v.23, p.441-444, 2001.

BAZZOLI, N.. Biologia reprodutiva do peixe-cachorro Acestrorhynchus lacustris (Reinhardt, 1874) (Characidae, Acestrorhynchinae) da Represa de Três Marias, Rio São Francisco/MG. Dissertação (Mestrado em Morfologia) Universidade Federal de Minas Gerais, Belo Horizonte, 1985.

BRAGA, F. M. S.. Estudo entre o fator de condição e relação peso/comprimento para alguns peixes marinhos. Rev. Brasil. Biol., v.46, p.339-346, 1986.

BRAGA, F. M. S.; GENNARI-FILHO.. Contribuição para o conhecimento da reprodução de Moenkhausia intermedia (Characidae, Tetragonopterinae), na represa de Barra Bonita, rio Piracicaba, São Paulo. Naturalia, v.15, p.171-188, 1990.

BRAGA, L. G. T.; BORGHESI, R.; DAIRIKI, J. K.; CYRINO, J. E. P.. Trânsito gastrintestinal de dieta seca em Salminus brasiliensis. Pesquisa Agropecuaria Brasileira, Brasilia, DF, v.42, n.1, p.131-134, 2007

COSTA, A. P. R.; ANDRADE D. R.; JUNIOR M. V. V.; SOUZA, G.. Indicadores quantitativos da biologia reprodutiva de fêmeas de piau-vermelho no Rio Paraíba do Sul, Pesq. agropec. bras. Brasília, v.40, p.789-795, 2005.

FEITOSA, L. A.; FERNANDES, R.; DA COSTA, R. S.; GOMES, L. C.; AGOSTINHO, A. A.. Parâmetros populacionais e simulação do rendimento por recruta de Salminus brasiliensis (Cuvier, 1816) do Alto rio Paraná. Acta Sci. Biol. Sci., Maringá, v.26, p.317-323, 2004.

HERMES-SILVA, S.. Distribuição espacial e temporal do ictioplâncton no alto rio Uruguai. Dissertação (Mestrado em aquicultura) - Universidade Federal de Santa Catarina, Florianópolis, 2003.

HONJI, R. M.. Caracterização endócrina durante o ciclo reprodutivo da tabarana Salminus hilarii (Characiformes: Characidae), em três ambientes distintos: natural, impactado e cativeiro. Dissertação (Mestrado em Ciências, na área de Fisiologia) - Instituto de Biociências da Universidade de São Paulo, 2007

MACHADO, C.. Aspectos reprodutivos do dourado Salminus brasiliensis (Cuvier, 1816) (TELEOSTEI, CHAEACIDAE) na Região do Alto rio Uruguai, Brasil. Dissertação (Mestrado em aquicultura) - Universidade Federal de Santa Catarina, 2003.

MAZZONI, R.; SILVA. A. P. F.. Aspectos da história de vida de Bryconamericus microcephalus (Miranda Ribeiro) (Characiformes, Characidae) de um riacho costeiro de Mata Atlântica, Ilha Grande, Rio de Janeiro, Brasil, Rev. Bras. Zoll. Curitiba, v.23, p.228-233, 2006.

MORAIS-FILHO, M. B.; SCHUBART, O.. Contribuição ao estudo do dourado (Salminus maxillosus) do rio Mogi Guaçu. Publ. Min. Agric., São Paulo, n.114, 1955.

MOREIRA, R. G.. Esteroides gonadais, proteínas, lipídios plasmáticos e hepáticos em relação ao ciclo reprodutivo do dourado (Salminus maxillosus) (Pisces, Characidae) de ambiente natural. Tese (Doutorado em Fisiologia) Universidade de São Paulo, 1999.

OLARTE, D. R.; TAPHORN, B. D. C.. Abundance, feeding and reproduction of Salminus sp. (Pisces: Characidae) from mountain streams of the Andean piedmontin Venezuela. Neotropical Ichthyology, Porto Alegre, v.4, p.73-79, 2006.

RESENDE, E. K.. Pulso de inundação: processo ecológico essencial à vida no Pantanal. Embrapa Pantanal Documentos, 2008.

VAZZOLER, A. E. A.. Biologia da reprodução de peixes teleósteos: Teoria e prática. Maringá: Eduem, 1996.

VILLARES JUNIOR, G. A.; GOMIERO, L. M.; GOITEIN. R. Relação peso-comprimento e fator de condição de Salminus hilarii Valenciennes 1850 (Osteichthyes, Characidae) em um trecho dabacia do rio Sorocaba, Estado de São Paulo, Brasil. Acta Sci. Biol. Sci. Maringá, v.29, p.407-412, 2007.

ZANIBONI-FILHO, E.; NUÑER, A. P. O.; FRACALOSSI, D.; TATAJE, D. A. R.; HERMES-SILVA, S.; WEINGARTNER, M.. Relatório técnico parcial do monitoramento da ictiofauna do reservatório da UHE Itá. Florianópolis: LAPAD, 2005.

A CBPC - Companhia Brasileira de Produção Científica (CNPJ: 11.221.422/0001-03) detém os direitos materiais desta publicação. Os direitos referem-se à publicação do trabalho em qualquer parte do mundo, incluindo os direitos às renovações, expansões e disseminações da contribuição, bem como outros direitos subsidiários. Todos os trabalhos publicados eletronicamente poderão posteriormente ser publicados em coletâneas impressas sob coordenação da Sustenere Publishing, da Companhia Brasileira de Produção Científica e seus parceiros autorizados. Os (as) autores (as) preservam os direitos autorais, mas não têm permissão para a publicação da contribuição em outro meio, impresso ou digital, em português ou em tradução. 